\title{
Energy Efficient MAC Protocol \& Radio Energy Model for AODV \& Bellman Ford Routing Protocol
}

\author{
Manish Uzzwal ${ }^{1}$, Rajan Sharma ${ }^{2}$ \\ ${ }^{1}$ University Institute of Engineering, Chandigarh University, Punjab, India \\ ${ }^{2}$ Department of Electronics \& Communication, Chandigarh University, Punjab, India
}

\begin{abstract}
Sensor Network devices have limited battery resources consumed by radio communication. Power saving is a critical issue for network devices. This paper is subjected to Adhoc on Demand Distance Vector $(A O D V)$ \& Bellman Ford Routing protocol performance with IEEE 802.11 \& IEEE 802.15.4 MAC Protocol in random topology for 50 nodes \& evaluated its performance. We investigated the energy consumption in transmit mode, receive mode, idle mode \& residual battery by changing the MAC Protocol \& Radio Energy Model through QualNet 6.1.The main interest of this paper is to identify the energy efficient Radio Energy Model \& MAC Protocol.
\end{abstract}

Keywords: AODV, Bellman Ford, IEEE 802.11, IEEE 802.15.4, MAC Protocol, QualNet 6.1, Radio Energy Model.

\section{Introduction}

Energy efficiency is a basic requirement for network operations [1]. Sensor networks are constrained by limited battery power resources, of which the primary consumer is the radio. Sensor nodes play different communication roles and consequently consume different amounts of energy. Since in wireless node the battery energy is finite \& a node can only transmit a finite number of bits. The maximum number of bits that can be sent is defined by the total battery energy divided by the required energy per bit. Thus nodes carrying a heavier burden of communication may prematurely deplete their batteries and partition the network such that other nodes cannot communicate despite having energy remaining. The nodes play a critical role in maintaining connectivity and delivering the sensor data are those at greatest risk for depletion. A major power consuming component of a sensor node is the radio which is controlled by the MAC protocol. Therefore an efficient MAC protocol increases the lifetime of a sensor network to a great extent[2].

\section{Related Work}

V.K.Taksande et al. [3] simulated AODV Protocol with IEEE 802.11 MAC for Chain Topology in MANET. This paper investigated the performance metrics namely throughput by varying network size up to 55 nodes through NS-2 simulation. Almost always the network protocols were simulated as a function of mobility not as a function of network density in chain topology. The main interest of this paper is to test the ability of AODV routing protocol to react on chain network topology as number of nodes changes..

Deepti Gupta et al. [4] simulated the Performance Evaluation of WSN Routing Protocols for MICA and MICAz using Different Radio Models. This paper has been made to demonstrate the behavior of routing protocols in presence of realistic radio models. Moreover the simulation results show that the energy consumption decreases and lifetime of sensor networks increase when MICA motes are used instead of the MICAz motes. Hence MICA motes could be used to improve the sensor network performance in real time.

Mario Di Francesco et al. [5] Reliability and Energy efficiency in IEEE 802.15.4/ZigBee Sensor Networks: An Adaptive and Cross-layer Approach. This paper propose an adaptive and cross layer framework for reliable and energy efficient data collection in WSNs based on the IEEE 802.15.4/ZigBee standards. The framework involves an energy aware adaptation module that captures the application's reliability requirements and autonomously configures the MAC layer based on the network topology and the traffic conditions in order to minimize the power consumption.

\section{Mac Protocols}

IEEE 802.11 is the first wireless LAN (WLAN) standard proposed in 1997 [6]. The medium access mechanism, called the Distributed Coordination Function is basically a Carrier Sense Multiple Access with Collision Avoidance mechanism (CSMA/CA). A station wanting to transmit senses the medium. If the medium is busy then it defers. If the medium is free for a specified time (called Distributed Inter Frame Space DIFS in the standard), then the station is allowed to transmit. The receiving station checks the CRC(Cyclic Redundancy Check) of the received packet and sends an acknowledgment packet(ACK). If the sender does not receive the ACK then it retransmits the frame until it receives ACK or is thrown away after a given 
number of retransmissions. According to the standard a maximum of seven retransmissions are allowed before the frame drops. In order to reduce the probability of two stations colliding due to not hearing each other which is well known as the "hidden node problem". The standard defines a Virtual Carrier Sense mechanism. A station wanting to transmit a packet first, transmits a short control packet called RTS (Request To Send) which includes the source destination and the duration of the intended packet and ACK transaction. The destination station responds (if the medium is free) with a response control packet called CTS (Clear to Send) which includes the same duration information. Obviously collisions are still possible because the efficiency of CSMA/CA depends on the sensing range of each node and the presence of a hidden station. In general the performances of CSMA/CA are strictly related to the network topology and the nodes density. The more nodes can hear each other the better quality of communication can be achieved avoiding collisions. Inevitably large latency times affect the efficiency of the system because before transmitting each station has to wait an unpredictable amount of time that mainly depends on the demands of users and topology of the network.

IEEE 802.15.4 MAC is a member of the IEEE 802 family but it does not mean that all the features of all the other IEEE 802 standards are included or even desired for this low rate, low duty cycle standard. The mission for this standard was to empower simple devices with a reliable, robust wireless technology that could run for years on standard primary batteries .By favoring low cost and low power IEEE 802.15.4 is enabling applications in the fields of industrial, agricultural, vehicular, residential and medical sensors and actuators. Until recently these applications could not make use of current wireless technologies or would have to use proprietary solutions (in most cases unidirectional) [7, 8]. The intent of IEEE 802.15.4 is to address applications where existing WPAN solutions are too expensive and the performance of a technology such as Bluetooth is not required. IEEE 802.15.4 LR-WPANs complement other WPAN technologies by providing very low power consumption capabilities at very low cost thus enabling applications that were previously impractical.

\subsection{AODV Routing Protocol}

\section{Routing Protocol}

Ad hoc On-Demand Distance Vector (AODV) [9] Routing is a routing protocol for mobile ad hoc networks (MANETs) and other wireless ad hoc networks. In AODV the network is silent until a connection is needed. At that point the network node that needs a connection broadcasts a request for connection. Other AODV nodes forward this message, and record the node that they heard it from, creating an explosion of temporary routes back to the needy node. When a node receives such a message and already has a route to the desired node, it sends a message backwards through a temporary route to the requesting node. The needy node then begins using the route that has the least number of hops through other nodes. Unused entries in the routing tables are recycled after a time. When a link fails a routing error is passed back to a transmitting node and the processes repeats. The advantage of AODV is that it creates no extra traffic for communication along existing links. Also distance vector routing is simple and doesn't require much memory or calculation. However AODV requires more time to establish a connection, and the initial communication to establish a route is heavier than some other approaches.

\subsection{Bellman Ford Routing Protocol}

Bellman Ford Routing Algorithm [10] also known as Ford Fulkerson Algorithm, is used as a distance vector routing protocol. Routers that use this algorithm have to maintain the distance tables, which tell the distances and shortest path to sending packets to each node in the network. The information in the distance table is always updated by exchanging information with the neighbouring nodes.

\section{Radio Energy Model}

The Radio Energy [11] model reads the energy consumption specifications of the radio. Where the specifications are defined by the configuration parameters. Which are the power supply voltage of the radio, electrical current load consumed in transmit, receive, idle \& sleep modes.

Generic Radio Energy Model is used for the estimation of energy consumption for the radios with common modulation schemes \& common class of amplifiers. The model can estimate energy consumption in transmitter for the case of continuous transmits power level. The second is Mica Mote Radio Energy Model which is a radio specific energy model which is preconfigured with the specification of power consumption of Mica Mote 


\subsection{Simulation Scenario}

\section{Simulation Environment}

Here we give the emphasis for the valuation of energy performance of Ad hoc routing protocol AODV \& Bellman Ford with varying the MAC Protocol \& Radio Energy Model. The simulation has been performed using network simulator QualNet 6.1 over Windows platform. Qualnet is a discrete event simulator [12].It is equally capable of simulating various wired or wireless scenarios from simple to complex conditions. In the simulation model there are 50 nodes and all of these are connected to one wireless stations placing within a $500 \mathrm{~m} \times 500 \mathrm{~m}$ area. Simulation time we have used is $100 \mathrm{~s}$. All the nodes we have assumed as fixed one .Shown in Fig. 1 .The performance of AODV \& Bellman Ford Routing Protocol is evaluated by varying the MAC protocol \& Radio energy model. Keeping the network size constant. The numbers of constant bit rate (CBR) connections are 10.The entire connection set up has been done randomly. In this we use the concept of RFD and FFD.Then we further makes the coordinator and PAN Coordinator. The simulation parameters used in this valuation have been mentioned in Table I.

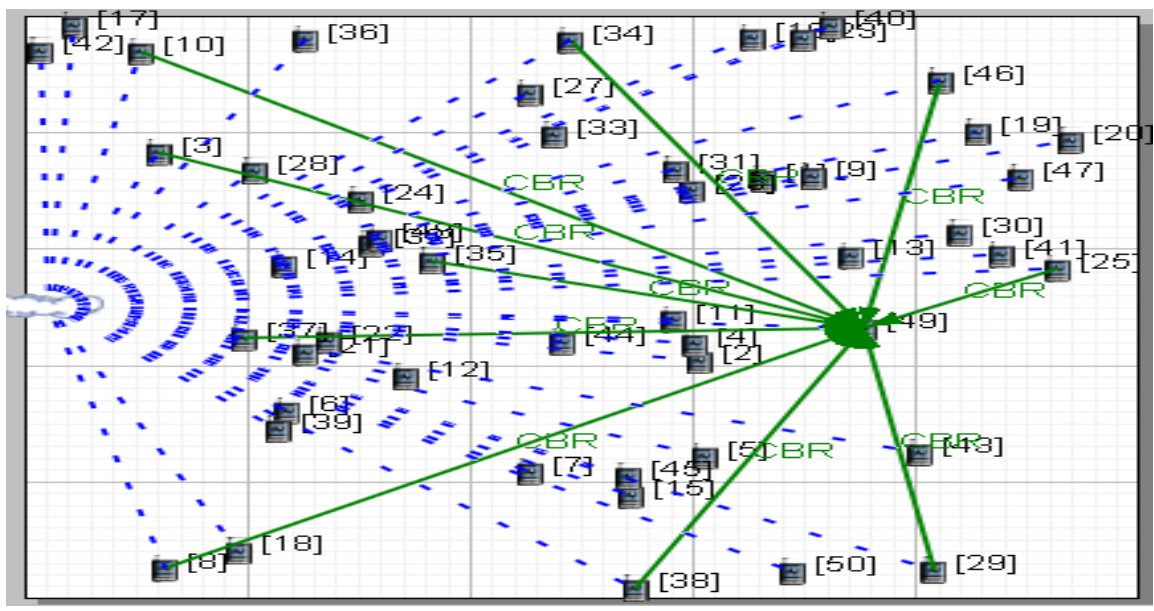

Fig. 1 Simulation Scenario

Table 1. Parameter Values for Simulation

\begin{tabular}{|l|l|}
\hline Simulation Simulator & QualNet 6.1 \\
\hline MAC Layer Protocol & IEEE 802.11 and IEEE 802 15.4 \\
\hline Routing Protocol & AODV,Bellman Ford \\
\hline Number of Nodes & 50 \\
\hline Simulation Duration & 100 seconds \\
\hline Simulation Area & $500 \mathrm{~m}$ x 500 m \\
\hline Network Protocol & IPV4 \\
\hline Movement Model & Random Topology \\
\hline Transmission Power(dBm) & 3 \\
\hline Battery Model & Linear \\
\hline Altitude Range(meter) & 1500 above sea level \\
\hline Number of Channels & 1 \\
\hline Traffic Type & CBR(UDP) \\
\hline
\end{tabular}

\section{Simulation Results \& Observation}

The simulation results are shown in the following section in the form of Column graph. The performance of AODV \& Bellman Ford Routing Protocol is based on the varying the MAC Protocol \& Radio Energy model in Random Topology is done on parameters like energy consumed in transmit mode, receive mode, idle mode \& average battery residual. 
"Fig. 2" highlights the relative performance of AODV Routing Protocol performance with IEEE 802.11 \& IEEE 802.15.4 MAC Protocol with Generic \& Mica Mote Radio Energy Model. From the figure it is observe that AODV Routing Protocol with MAC Protocol IEEE 802.11 \& Mica Mote Radio Energy Model consumes $0.001366 \mathrm{mWh}$ energy. Which is the least energy consumed in Transmit Mode.

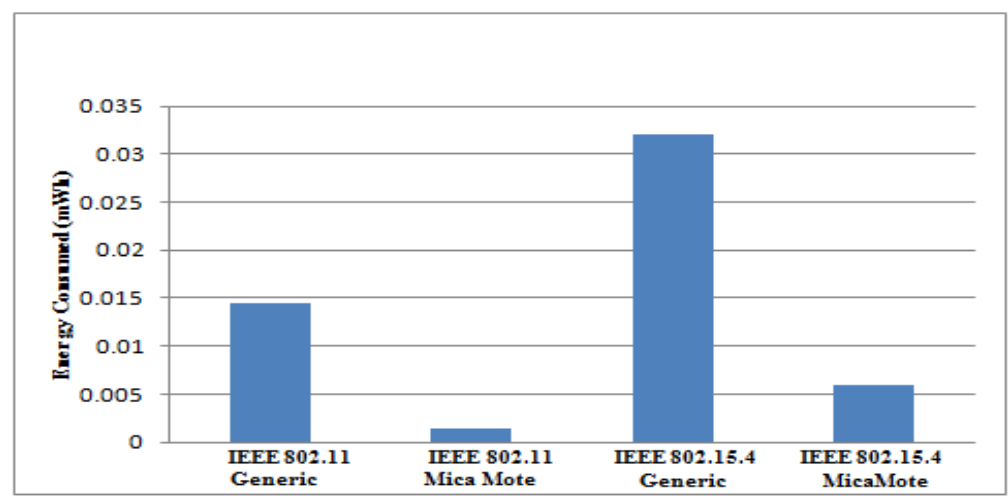

Fig. 2 Transmit Mode

"Fig. 3" highlights the relative performance of AODV Routing Protocol performance with IEEE 802.11 \& IEEE 802.15.4 MAC Protocol with Generic \& Mica Mote Radio Energy Model. From the figure it is observe that AODV Routing Protocol with MAC Protocol IEEE 802.11 \& Mica Mote Radio Energy Model consumes $0.013375 \mathrm{mWh}$ energy. Which is the least energy consumed in Receive Mode.

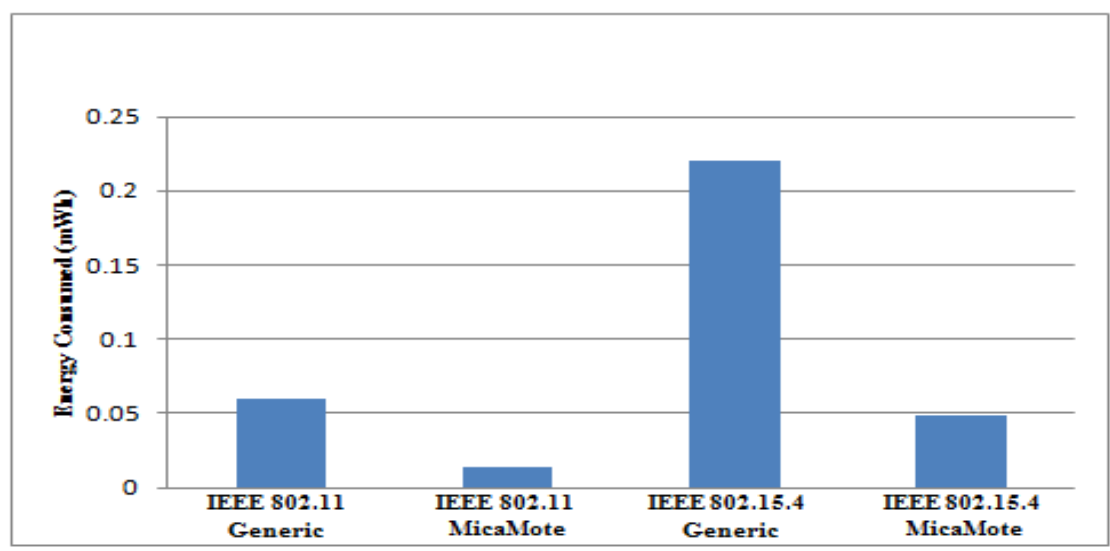

Fig. 3 Receive Mode

"Fig. 4" highlights the relative performance of AODV Routing Protocol performance with IEEE 802.11 \& IEEE 802.15.4 MAC Protocol with Generic \& Mica Mote Radio Energy Model. From the figure it is observe that AODV Routing Protocol with MAC Protocol IEEE 802.15.4 \& Mica Mote Radio Energy Model consumes $0.145239 \mathrm{mWh}$ energy. Which is the least energy consumed in Idle Mode.

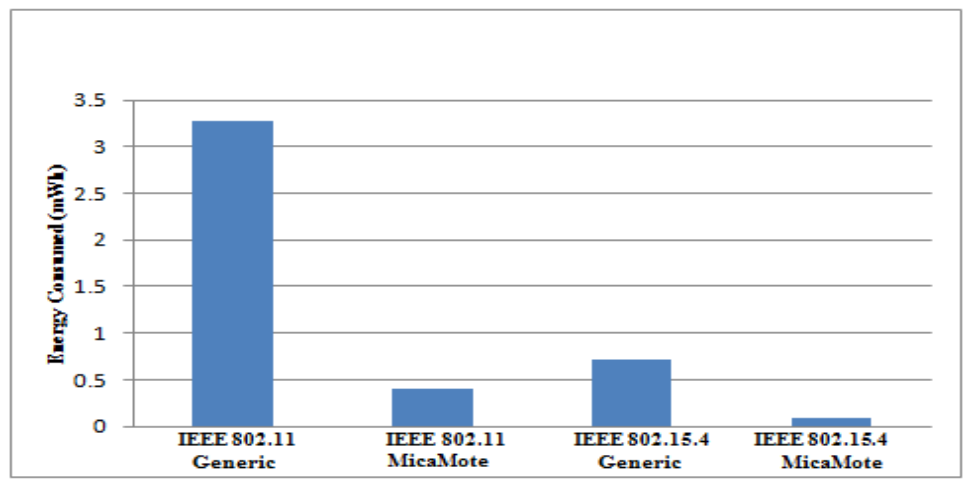

Fig. 4 Idle Mode 
"Fig. 5" highlights the relative performance of AODV Routing Protocol performance with IEEE 802.11 \& IEEE 802.15.4 MAC Protocol with Generic \& Mica Mote Radio Energy Model. From the figure it is observe that AODV Routing Protocol with MAC Protocol IEEE 802.15.4 \& Mica Mote Radio Energy Model retains $119.951 \mathrm{mAhr}$ Residual Battery. This is the maximum residual battery after the complete simulation of the network.

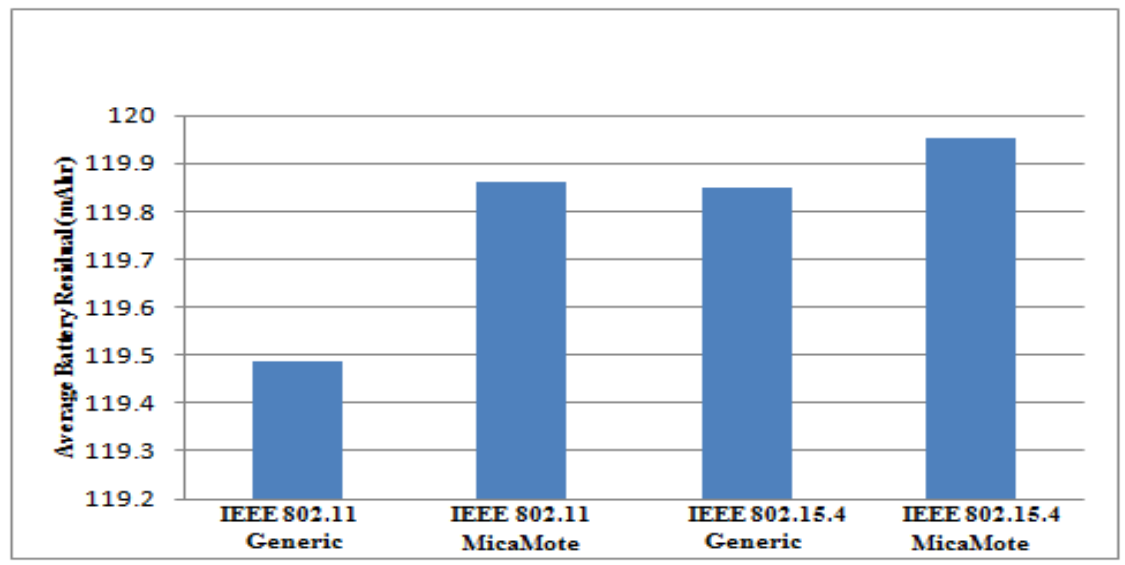

Fig. 5 Residual Battery

"Fig. 6" highlights the relative performance of Bellman Ford Routing Protocol performance with IEEE 802.11 \& IEEE 802.15.4 MAC Protocol with Generic \& Mica Mote Radio Energy Model. From the figure it is observe that Bellman Ford Routing Protocol with MAC Protocol IEEE 802.11 \& Mica Mote Radio Energy Model consumes $0.002984 \mathrm{mWh}$ energy. Which is the least energy consumed in Transmit Mode.

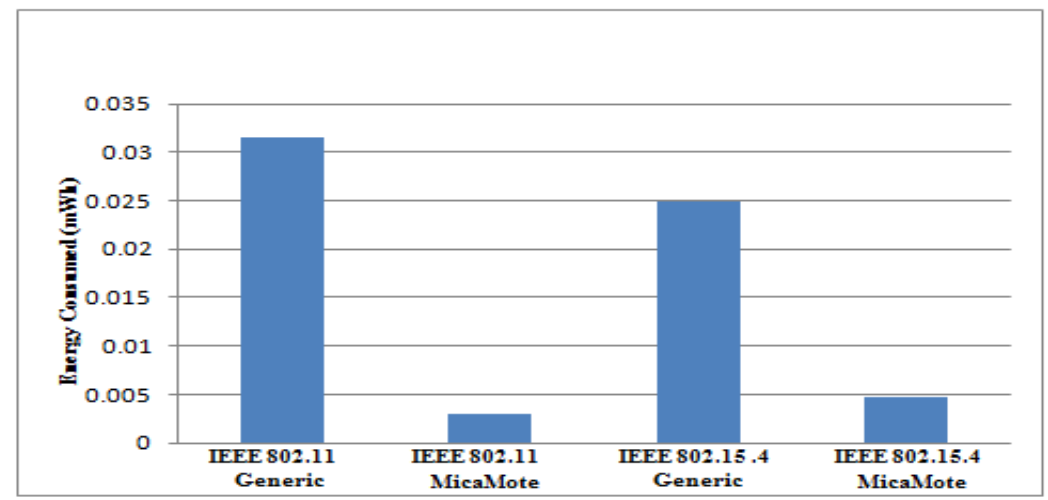

Fig. 6 Transmit Mode

"Fig. 7" highlights the relative performance of Bellman Ford Routing Protocol performance with IEEE 802.11 \& IEEE 802.15.4 MAC Protocol with Generic \& Mica Mote Radio Energy Model. From the figure it is observe that Bellman Ford Routing Protocol with MAC Protocol IEEE 802.11 \& Mica Mote Radio Energy Model consumes $0.029547 \mathrm{mWh}$ energy. Which is the least energy consumed in Receive Mode

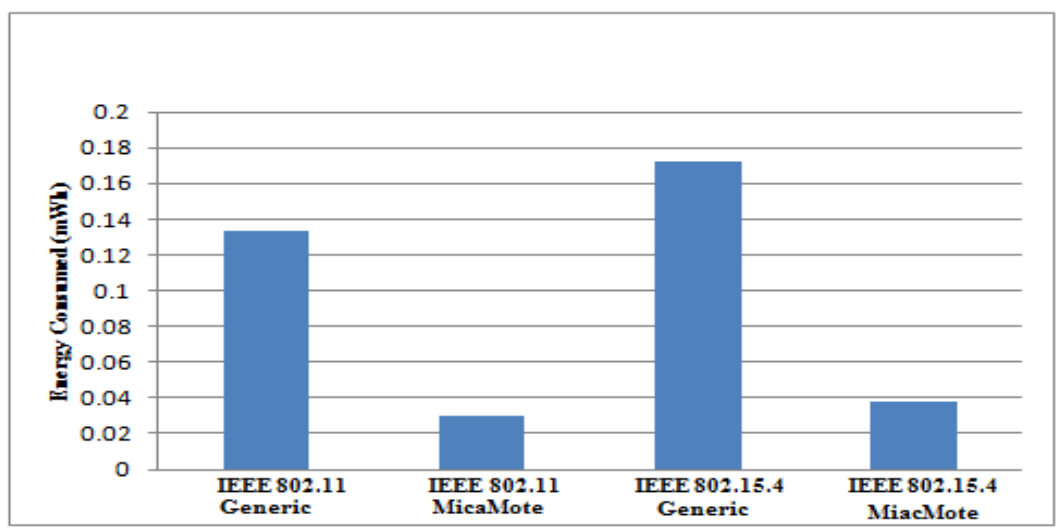

Fig. 7 Receive Mode 
"Fig. 8" highlights the relative performance of Bellman Ford Routing Protocol performance with IEEE 802.11 \& IEEE 802.15.4 MAC Protocol with Generic \& Mica Mote Radio Energy Model. From the figure it is observe that Bellman Ford Routing Protocol with MAC Protocol IEEE 802.15.4 \& Mica Mote Radio Energy Model consumes $0.091231 \mathrm{mWh}$ energy. Which is the least energy consumed in Idle Mode.

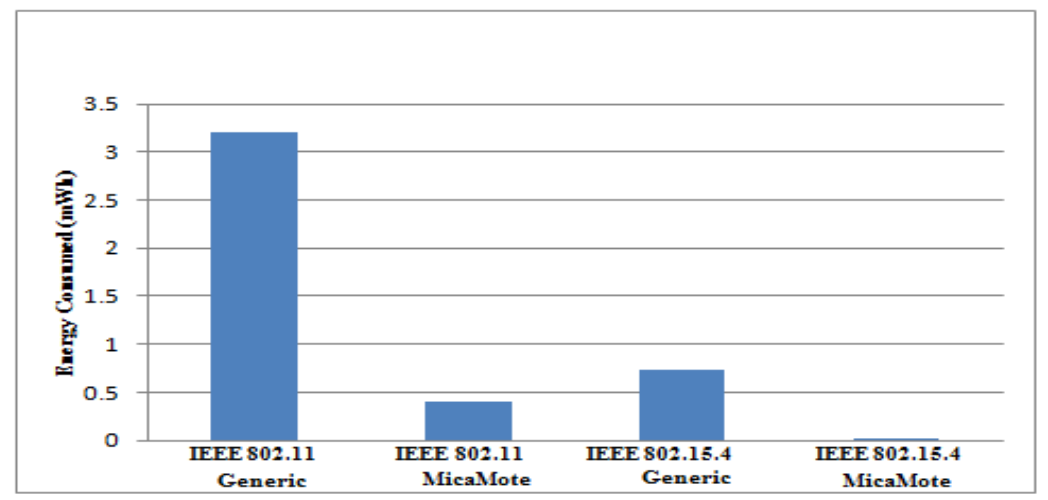

Fig. 8 Idle Mode

"Fig. 9" highlights the relative performance of Bellman Ford Routing Protocol performance with IEEE 802.11 \& IEEE 802.15.4 MAC Protocol with Generic \& Mica Mote Radio Energy Model. From the figure it is observe that Bellman Ford Routing Protocol with MAC Protocol IEEE 802.15.4 \& Mica Mote Radio Energy Model retains 119.946 mAhr Residual Battery. This is the maximum residual battery after the complete simulation of the network.

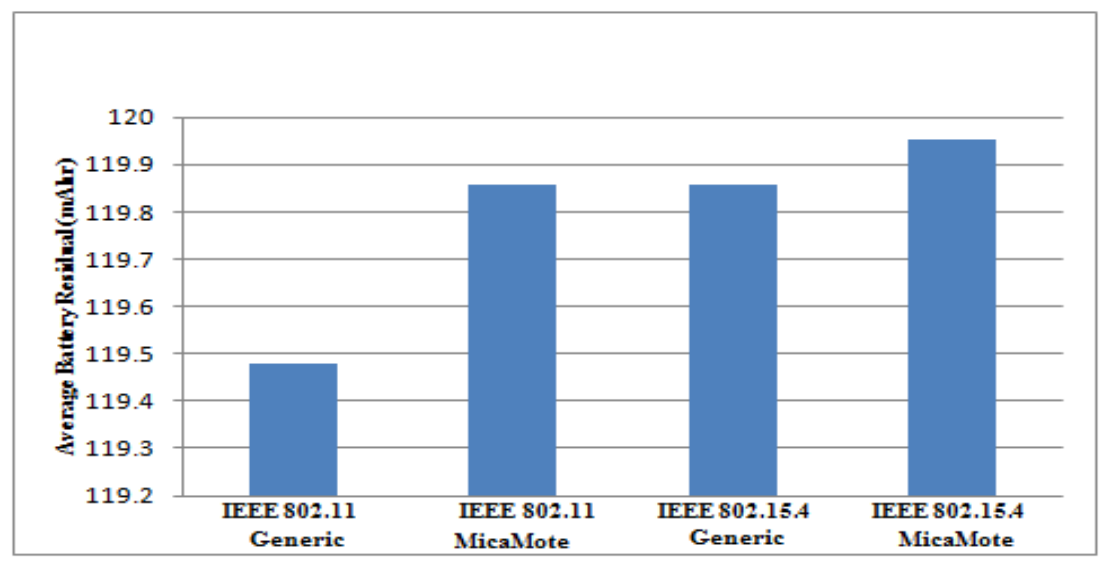

Fig. 9 Residual Battery

\section{Conclusions}

In this presented simulation work, AODV \& Bellman Ford Routing Protocol are evaluated for efficient MAC protocol \& Radio Energy Model in Random Topology. As we vary the MAC Protocol \& Radio Energy Model for performing the simulation of AODV \& Bellman Ford the energy consumed in Transmit Mode, Receive Mode, Idle Mode \& Residual Battery changes. As a result of our studies we conclude that IEEE 802.15.4 MAC Protocol with Mica Mote Radio Energy Model exhibits least average energy consumption \& maximum residual battery in random topology.

Considering the overall performance of AODV \& Bellman Ford Routing Protocol the energy efficient MAC Protocol \& Radio Energy Model is IEEE 802.15.4 MAC Protocol \& Mica Mote Radio Energy Model.

\section{References}

[1] Xu, Y., Hendemann, J., \& Estrin, D. (2000). "Adaptive energy- conserving routing for multihop ad hoc networks". Technical report TR-2000-527, USC/Information Sciences Institute.

[2] I. Demirkol, C. Ersoy, F. Alagoz, "MAC Protocols for Wireless Sensor Networks: A Survey", accepted by IEEE Communications, 2005 .

[3] V.K.Taksande and Dr.K.D.Kulat, "An Extensive Simulation Analysis of AODV Protocol with IEEE 802.11 MAC for Chain Topology in MANET", International Journal of Internet Computing, Volume-I, Issue-1, 2011.

[4] Deepti Gupta and Ajay K Sharma, "On Performance Evaluation of WSN Routing Protocols for MICA and MICAz using Different Radio Models”, International Journal of Energy, Information and Communications Vol. 2, Issue 4, November, 2011. 
[5] Mario Di Francesco, Giuseppe Anastasi, Marco Conti, Sajal K .Das and Vincenzo Neri, "Reliability and Energy-efficiency in IEEE 802.15.4/ZigBee Sensor Networks: An Adaptive and Cross-layer Approach", Ieee Journal On Selected Areas In Communications, Vol. 29, No. 8, September 2011.

[6] IEEE 802.11 - Wireless LAN media access control (MAC) and physical layer (PHY) specifications. 1999

[7] Rafidah Ahmed, Othman Sidek and Shukri Korakkottil Kunhi Mohd. (2009), "Development of CRC Block on FPGA for Zigbee Standards," IEEE Trans. Industrial Electronics, CEDEC Engineering Campus, Malaysia, 2009

[8] P Mohana, S Radha (2009), "Realization of MAC Layer Functions of ZigBee Protocol Stack in FPGA", International Conference on "Control Automation, Communication and energy conservation -2009", 4th-6th, June 2009

[9] Luke Klein - Bamdt- Wireless Communication Technology group -A Quick Guide To AODV http://w3.antd.nist.gov/wctg/aodv_kernel/aodv_guide.pdf

[10] A.Boomarani Malany ,V.R.Sarma ,Dhulipala and RM.Chandrasekaran, "Throughput and Delay Comparison of MANET Routing Protocols", Int. J. Open Problems Compt. Math., Vol. 2, No. 3, September 2009

[11] QualNet documentation, QualNet 6.1 Model Library:Wireless, http://www.scalablenetworks.com

[12] Scalable Network Technologies, "Qualnet simulator", Software Package, 2003. http:// www.scalable-networks.com 